\title{
Idiopathic carpal tarsal osteolysis (ICTO) with additional elbow involvement
}

\author{
Farzanah Ismail • Craig Muller • Ruan Goller • \\ Zarina I. Lockhat $\cdot$ Savvas Andronikou
}

Received: 9 April 2011 /Revised: 18 June 2011 / Accepted: 4 July 2011 /Published online: 9 August 2011

(C) ISS 2011

\begin{abstract}
Answer
Idiopathic carpal tarsal osteolysis (ICTO) with additional elbow involvement.
\end{abstract}

\section{Discussion}

ICTO is a rare congenital disorder characterized by bone resorption affecting mainly the carpal and tarsal bones [1]. Previously it was known as idiopathic multicentric osteolysis and was first described in 1838 by this term [2]. Tyler et al. also preferred the term idiopathic multicentric osteolysis (1976) as this encompassed the wide array of descriptive terminology used to describe this condition in

The case presentation can be found at doi:10.1007/s00256-011-1233-0.

The authors declare that no funding or grants have been received.

F. Ismail $\cdot$ C. Muller $\cdot$ R. Goller $\cdot$ Z. I. Lockhat $\cdot$ S. Andronikou University of Pretoria,

Pretoria, South Africa

C. Muller

e-mail: drcraigmuller@gmail.com

R. Goller

e-mail: ruan.goller@up.ac.za

Z. I. Lockhat

e-mail: zarina.lockhat@up.ac.za

S. Andronikou

e-mail: docsav@mweb.co.za

F. Ismail $(\bowtie)$

Department of Radiology, Level 5, Steve Biko Academic

Hospital, University of Pretoria,

Voortrekker Road, Gezina,

Pretoria, South Africa 0002

e-mail: drfismail@yahoo.com the literature [3]. The term idiopathic carpal tarsal osteolysis was first used by Froelich and Coerret in 1937 [1, 4]. It is also known as multicentric carpal-tarsal osteolysis with and without nephropathy, and the association of renal failure was first described by Marie et al. in 1951 [2, 4].

The condition may occur sporadically or is inherited as an autosomal dominant or recessive condition [4]. Knowledge about the genetics of this condition is still evolving. It was divided into five subtypes by Hardegge et al. in 1985. Type I is inherited as an autosomal dominant condition and is infrequently associated with renal involvement. Type II is inherited as autosomal recessive with no renal involvement. Type III is sporadic with frequent renal involvement [2]. Type IV is also known as Gorhams disease and is unicentric and caused by haemangiomatosis. Type $\mathrm{V}$ is also known as Winchester syndrome and is associated with a different clinical presentation, corneal clouding, contractures, and skin lesions $[2,3]$.

Patients with ICTO present in early childhood with progressive acromelic joint pain, limitation of movement, swelling, and deformity $[2,4]$. The onset of disease usually begins around age 2 or 3 . Laboratory findings are normal with no evidence of inflammatory, metabolic, or autoimmune diseases $[2,4]$. This was the case with our patient.

The radiologic findings are characteristic with osteolysis of the carpal and tarsal bones and adjacent tubular bones [2]. The involvement of the adjacent metacarpals is described as "sucked candy" [3]. Osteolysis of the distal ulna (more than the radius) causes ulna deviation of the hand. Similar findings in the feet cause clubfoot deformity [2]. In this case there was asymmetry of the involvement of the hands. The changes on the right side were much more pronounced with the sucked candy appearance of the proximal metacarpals in contrast to the truncated appearance on the opposite sides. In contrast there was more 
dramatic involvement of the MCP joints on the left than the right. Both Carmichael and McDonald et al. described cases with elbow involvement. This was the case with our patient as well $[1,4]$.

Our patient was a sporadic presentation without current renal involvement (renal involvement is frequently associated with the sporadic type). Bennett et al. have found that bone involvement precedes renal involvement by 10 15 years, hence the need for long term follow-up of renal function [5]. In a patient with sporadic ICTO, Connor et al. found that the renal lesion was focal segmental glomerular sclerosis in early and subsequent renal biopsies. This patient was successfully treated with cyclosporin A. Proteinuria was the first sign of renal dysfunction [6].

The diagnosis of ICTO can only be made once other differential diagnoses such as juvenile idiopathic arthritis (JIA), trauma, inflammation, avascular necrosis, and hyperparathyroidism are excluded [4]. ICTO presents initially with joint stiffness, which progresses to hypermobility and a progressive decrease in pain. It stabilizes by age 20-30. This may aid in the exclusion of JIA, which demonstrates progressive signs and symptoms [2].
Treatment of ICTO is mainly supportive and may include physiotherapy, occupational therapy, and the use of orthotics and prosthetics.

\section{References}

1. Carmichael KD, Launikitis RA, Kalia A. The orthopaedic and renal manifestations of idiopathic carpal tarsal osteolysis. J Pediatr Orthop. 2007;16:451-4.

2. Faber MR, Verlaak R, Fiselier TJW, Hamel BCJ, Franssen MJAM, Gerrits GPJM. Inherited multicentric osteolysis with carpal tarsal localisation mimicking juvenile idiopathic arthritis. Eur J Pediatr. 2004;163:612-8.

3. Tyler T, Rosenbaum HD. Idiopathic multicentric osteolysis. Am J Roentgenol. 1976;126:23-31.

4. McDonald K, Toms AP, Armon K, Johnson K, Marshall TJ. Carpal-tarsal osteolysis with elbow involvement. Skeletal Radiol. 2007;36:1097-1101.

5. Bennett WM, Houghton DC, Beals RC. Nephropathy of idiopathic multicentric osteolysis. Nephron. 1980;25:134-8.

6. Connor A, Highton J, Hung NA, Dunbar J, MacGinley R, Walker R. Multicentric carpal-tarsal osteloysis with nephropathy treated successfully with cyclosporine A: a case report and literature review. Am J Kidney Dis. 2007;50:649-54. 